\title{
System of Soil Moisture Monitoring and Water-Saving Irrigation in Hulun Buir Based on Internet of Things and Neural Network
}

\author{
Xiaobo Hu ${ }^{a}$, Chaoyuan Cui ${ }^{b,}{ }^{*}$, Shu Yan ${ }^{c}$ \\ Hefei Institutes of Physical Science, Chinese Academy of Sciences, Hefei \\ 230031, China \\ a812762995@qq.com, b cycui@iim.ac.cn, c2282374449@qq.com
}

Keywords: soil moisture monitoring, water-saving irrigation, Internet of Things, Neural Network, Wireless Sensor Network, GM $(1,1)$.

\begin{abstract}
The soil moisture node and water-saving irragation node distribute in the farmland constituted Wireless Sensor Network(WSN) of system via communication technology of Internet Of Things(IOT) like ZigBee,3G/4G and so on.Intelligent sensor of soil moisture node collect soil moisture data and send the data to data-serving platform stored through WSN.Data-serving platform using soil moisture data sets build soil moisture prediction model by grey prediction method GM(1,1) of neural network.The model prediction result combine with crop varieties and irrigation threshod value to make policy of irrigated and irrigation amount,the policy send to water-saving irriagtion nodes as instruction text via WSN.The state and irrigation amount of the node will also feed back to data-serving paltform via WSN.Experiments and trial operation shows that this system can increase crop yield by $11.6 \%$ and saving water $78 \mathrm{~m}^{3}$ per mu, and system is very stable,easy to maintain,good expansibility,low cost and high degree of automation.
\end{abstract}

\section{Introduction}

Hulun Buir has rich resources of agriculture and livestock,and has plenty of fertile black land,whick is suitable for cultivation.However,the arid climate, water scarce,low soil water content and seriously water leakage.The blind and rough production will aggravate the loss of the black soil layer and cause desertification in this area[1].The development of water-saving irrigation technology can effectively improve crop yield and reduce weathering and loss of the black soil layer.

Drip,sprinkler and inflitration irrigation have been quite mature after years development.However,the time of irrigation and irrigation amount depends mostly on farmers experience,and there is a certain randomness and blindness[2].In recent years,with the development of IOT and artificial intelligence technology provide new ideas and methods to improve the efficiency of water-saving irrigation and reduce the cost.

This paper based on the collected soil moisture data builded the soil moisture prediction model, and automatically adjust the irrigation volume according to the pediction result,crop species and irrigation thresholds[3,4].The system has been tested and operated in the Hulun Buir area.The results show that this system can save the maximum irrigation water on basis of alleviating crop drought and raising crop yield[5].

\section{System Design}

Using block design idea to design soil moisture collection node,water-saving irrigation node,data transmission interface and data-serving platform.System architecture is shown in Figure 1. 


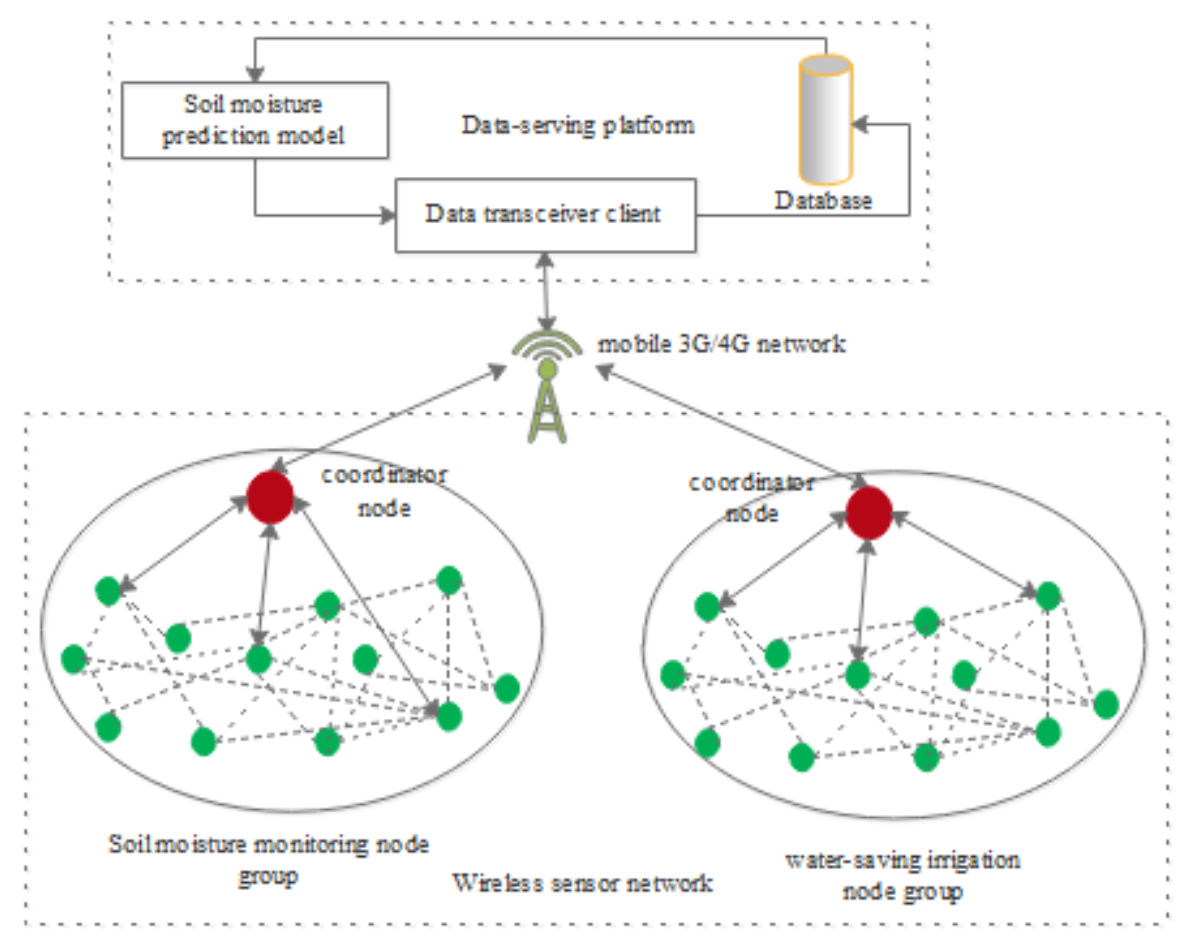

Fig.1 System architecture

Through ZigBee wireless communication network the soil moisture collection node and water-saving irrigation node formed node group.The data of each node aggregated in the coordinator node,then transmitted to data-serving paltform for storage and analysis by the mobile 3G/4G network[5,10].Each node group consitutes a WSN in the farmland.

The data from WSN are stored in platform database by the data transceiver client.Those data will be used to train and improve the soil moisture prediction model,which is builded by neural network.The model will predict soil moisture condition and trend of next week according to latest data from the WSN, and make a strategic decision on whether irrigation and irrigation volume.The decision translated to instruction text and sended to all water-saving irrigation node by data transceiver client .

\subsection{Design of Soil Moisture Monitoring Node}

Consideration of system cost,Selected domestic SMS-II-100 soil moisture sensor to measure soil moisture. The sensor has $-40^{\circ} \mathrm{C}$ to $80^{\circ} \mathrm{C}$ working temperature ,less than $3 \%$ measurement error, $5 \mathrm{~V}$ to $24 \mathrm{~V}$ working voltage, 0.5 senconds response time, $4 \mathrm{~mA}$ to $20 \mathrm{~mA}$ output signal .All those performance index is quite match system requirment.

TI CC2530 supports the latest Z-Stack 2007 protocol stack,built-in low-power enhanced 8051 microcontroller core,256KB programmable Flash,8KB RAM,RF front-end,timer,interrupt and memory controller,12 bit 8 to 1 analog-to-digital converter,21 universal IO port,etc.These features fully meet the needs of the system.This paper used CC2530 as the controller designed soil moisture monitoring node, water-saving irrigation node and coordinator node.

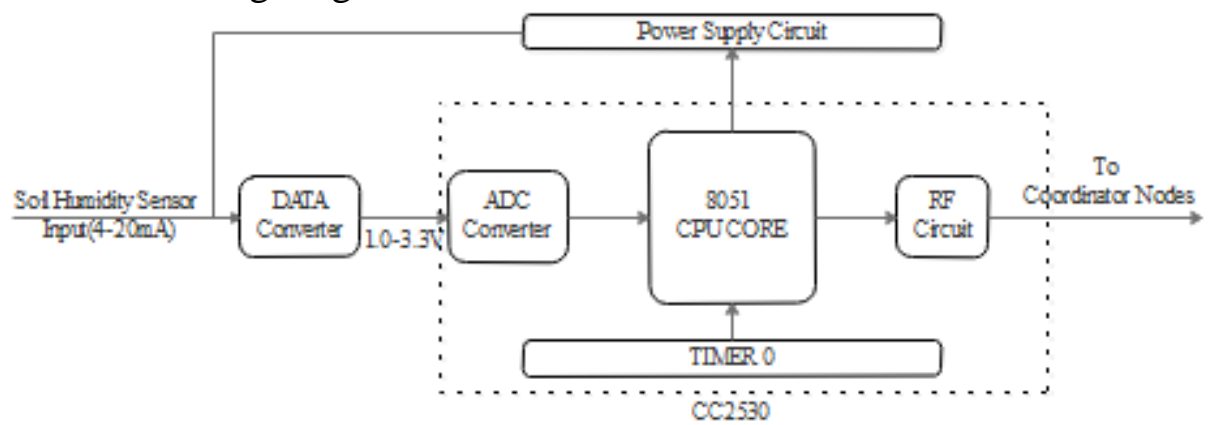

Fig.2 Soil moisture monitoring node constitution

The sleeping timer wake up CC2530 every hours for a data collection task,collecting ten soil moisture data,then takes the average and sends it to the coordinator node through RF circuit. 


\subsection{Design of Water-Saving Irrigation Node}

Water-saving irrigation nodes also uses CC2530 as the main controller chip to design hardware circuit.The node constitution as shown in following chart.

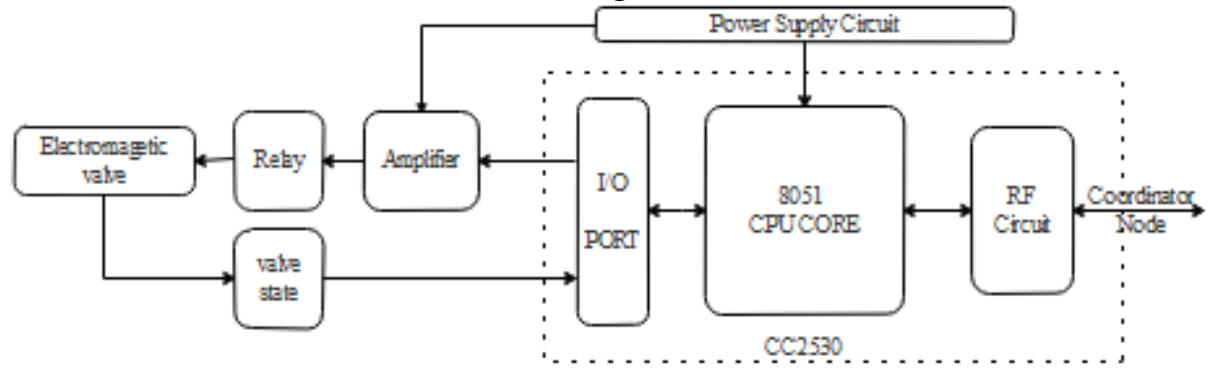

Fig.3 Water-saving irrigation node constitution

The coordinator node send instruction to each water-saving irrigation node in broadcasting mode.RF circuit received the instruction,then translated by CPU.According to the instruction the I/O port drive the electromagnetic valve in the water pipeline to irrigation through the amplifying circuit and the relay.The valve's state will also collected by CC2530,the send to coordinator node through RF circuit.

\subsection{Design of Coordinator Node}

Function of the coordinator node in this system includes establishment of WSN, ad hoc network,communication with the terminal nodes and data-serving platform[9].Select Shenzhen macro-electric H7710 3G DTU to communication with data-serving platform through mobile 3G network. The node circuit structure is shown as Figure 4.

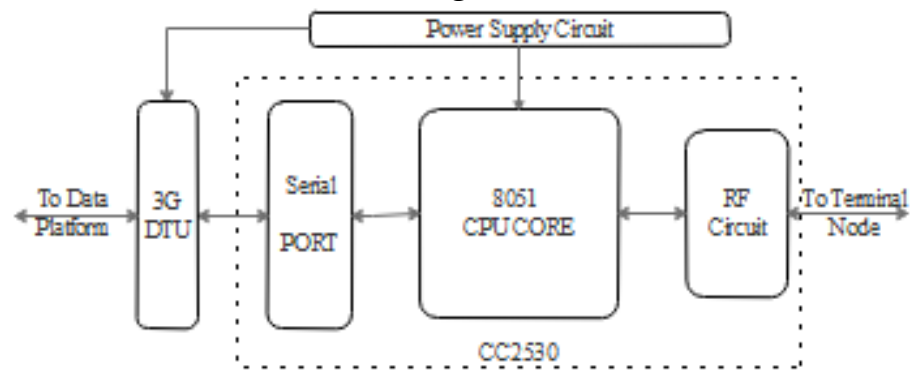

Fig.4 Coordinator node constructure

Due to the function difference between soil moisture monitoring nodes and water-saving irrigation nodes,in order to improve stability and scalability of the system,two wireless sensor networks are set up by different coordinate nodes.But the hardware circuit of two coordinator nodes are exactly the same.As shown in Figure 4,RF circuit receives data from terminal nodes,CPU processed the data and sends to DTU through the serial port, DTU sends data to sstorage and analysis in data-serving platform via mobile network. The result will feed back to coordinator nodes through mobile network and DTU,then distributed to each terminal nodes.

\subsection{Design of Data-Serving Platform}

The data-serving platform provides data storage,analysis and decision sevice for the system.It consists of three parts:MySQL database,soil moisture prediction model and data transceiver client.Data ransceiver client is used to communicate with coordinator and saving data from WSN to MySQL database. Soil moisture prediction model obtaine data from the database,then analysis and making decision on soil moisture. 


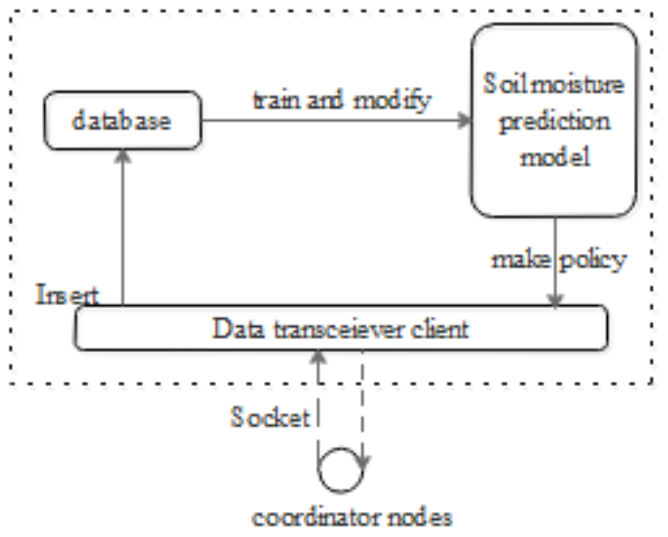

Fig.5 Data-serving client constitution

Data transceiver client is developed with Visual $\mathrm{C}++$ and communicates with coordinator node by Socket.Soil moisture prediction model is established by grey prediction model $\operatorname{GM}(1,1)$.The model algorithm is constructed in Java language.

\section{Method to Realized Soil Moisture Prediction Model}

Although the soil moisture is affected by many factors,the change regular is complex,but there is periodicity and regularity in general.It is feasible to establish the mode by using grey prediction method GM(1,1) to predict soil moisture in short term [7].

$\mathrm{GM}(1,1)$ soil moisture prediction model reflects first order differential function of soil moisture for time.The differential function is shown as follows:

$$
\frac{d x^{(1)}}{d t}+a x^{(1)}=u
$$

In which: ${ }_{x}{ }^{(1)}$ is accumulated generating sequence of soil moisture,t is time, a is development grey number, " is endogenous control grey number, a and " are unkown of the model.Build model is the process to figure out a and ".Build steps as following:

(1)Set up time series of soil moisture $x^{(0)}=\left(x^{(0)}(1), x^{(0)}(2), \cdots, x^{(0)}(n)\right)\left(x^{(0)}(n)\right.$ represented the soil moisture at $n$ time).

(2) Take smoothness test of ${ }_{x}^{(0)}$.

$$
(k)=\frac{x^{(0)}(k-1)}{x^{(0)}(k)} \quad k=2,3, \cdots, n
$$

If $\quad(k) \in\left[e^{\frac{-2}{n+1}}, e^{\frac{2}{n+1}}\right]$, the $x^{(0)}$ sequence can be used to established the model.

(3) Gernerate accumulative sequence $x^{(1)}(i)=\Sigma_{j} x^{(0)}(j) \quad i=1,2, \cdots, n$.

(4) Construct data matrix B and data vector Y.

$$
\begin{aligned}
& B=\left[\begin{array}{ccc}
-\frac{1}{2}\left[x^{(1)}(1)+x^{(1)}(2)\right] & 1 \\
-\frac{1}{2}\left[x^{(1)}(2)+x^{(1)}(3)\right] & 1 \\
\square & \\
-\frac{1}{2}\left[x^{(1)}(n-1)+x^{(1)}(n) 1\right.
\end{array}\right] \\
& Y=\left[x^{(0)}(2), x^{(0)}(3), \ldots, x^{(0)}(n)\right]
\end{aligned}
$$




$$
\hat{a}=\left[\begin{array}{l}
a \\
u
\end{array}\right]
$$

Because: $\frac{d_{x}^{(1)}}{d t}={ }_{x}^{(0)}, G M(1,1)$ model can be represented as $\quad$ y $=\hat{\text { в }}$ a .

(5)Figure out a , a .

$$
\hat{a}=\left(\begin{array}{l}
a \\
u
\end{array}\right)=\left(\begin{array}{llll}
B^{T} & B & -1 & \\
B & Y
\end{array}\right.
$$

(6)Get GM(1,1) model.

Bring a , u to formula 1 , get $\operatorname{GM}(1,1)$ model as following:

$$
\left.\hat{x}(1)(i+1)=\hat{(x}(0)(1)-\frac{u}{a}\right) e^{-a t}+\frac{u}{a}
$$

$\hat{x}^{(1)}(i+1)$ is accumulative value.Through following formula get prediction soil moisture value of next time.

$$
\hat{x}^{(0)}(i+1)=\hat{x}^{(1)}(i+1)-x^{(1)}(i)
$$

(7)Use the posterior error test method to examination model accuracy.

First calculate mean square error $s_{0}$ of ${ }_{x}^{(0)}(i)$.

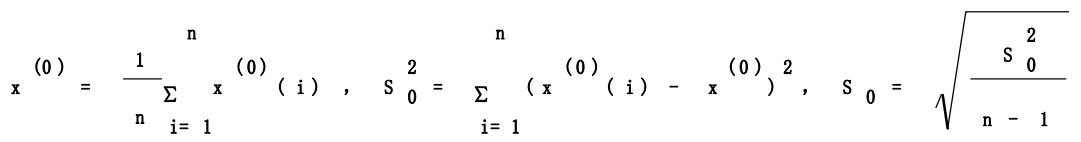

Then calculat the error between predicted value and actual value $\varepsilon^{0}(i)=x^{(0)}(i)-\hat{x}^{(0)}$ (i) , set up residual series.Figure out the series mean square error $s_{1}$.

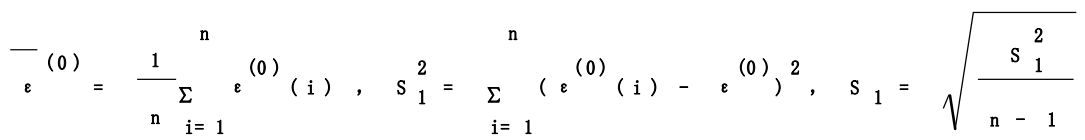

Figure out squre error ratio o and little error probability $\mathrm{p}$.

$$
c=\frac{S_{1}}{S_{0}}, p=\left\{\left|\varepsilon_{(0)}^{\left.(0)-\varepsilon^{(}\right)}\right|<0.64755 \square S_{0}\right\}
$$

If $c_{c}\left\langle 0_{3}\right.$ and ${ }_{p}>\rho_{.9}$, the model reaches qualified grade and can be used to predict the soil moisture.

\section{Application and Demonstration}

\subsection{Brief Introduction of the Demonstration Area}

The system is tested and demonstrated in Ewenk academician workstation of Hulun Buir Agriculture and Reclamation Group Company.The planting area of this station is about $200 \mathrm{mu}$, and crops are mainly forage, rape, wheat, oat and buckwheat, and water saving irrigation is drip irrigation.

\subsection{Selection of Experiment Area}

At the beginning of 2017, workstation unified consolidation of arable land at 200m length and $50 \mathrm{~m}$ width a plot according to breeding experiments requirment.We select six plot to applicate and demonstrate our system.Each plot is divided into ten grid units, and each grid deploys one soil moisture monitoring node.Considering that the drip pipe is required for pressure, we divided into five water-saving irrigation units.

\subsection{Sensor Deployment}

Crops like forage and wheat the maximum root can exceed one metre,but more than $80 \%$ of the root system is concentrated in the 0 centimeter to 40 centimeter soil layer.In order to the collection 
data can reflect water requirement of crops in the maximum extent,we deployed soil moisture sensor at 30 centimeter deepth in the soil layer.

\subsection{Analysis and Prediction}

Using collected soil moisture data from WSN in May and June,we set up GM(1,1) soil moisture prediction model,and in the beginning of July predicted the soil moisture,the contrast of prediction value and actual value as shown in Figure 6.Seen from the figure the model prediction is reliable,it can predict the trend of soil moisture in short time,fulfil the requirment of water-saving irrigation for the model.The model also has obvious hysteresis, which is determined by the characteristics of modeling.The maxium error occurred in July 6th,due to a heavy rainfall happened in the day.

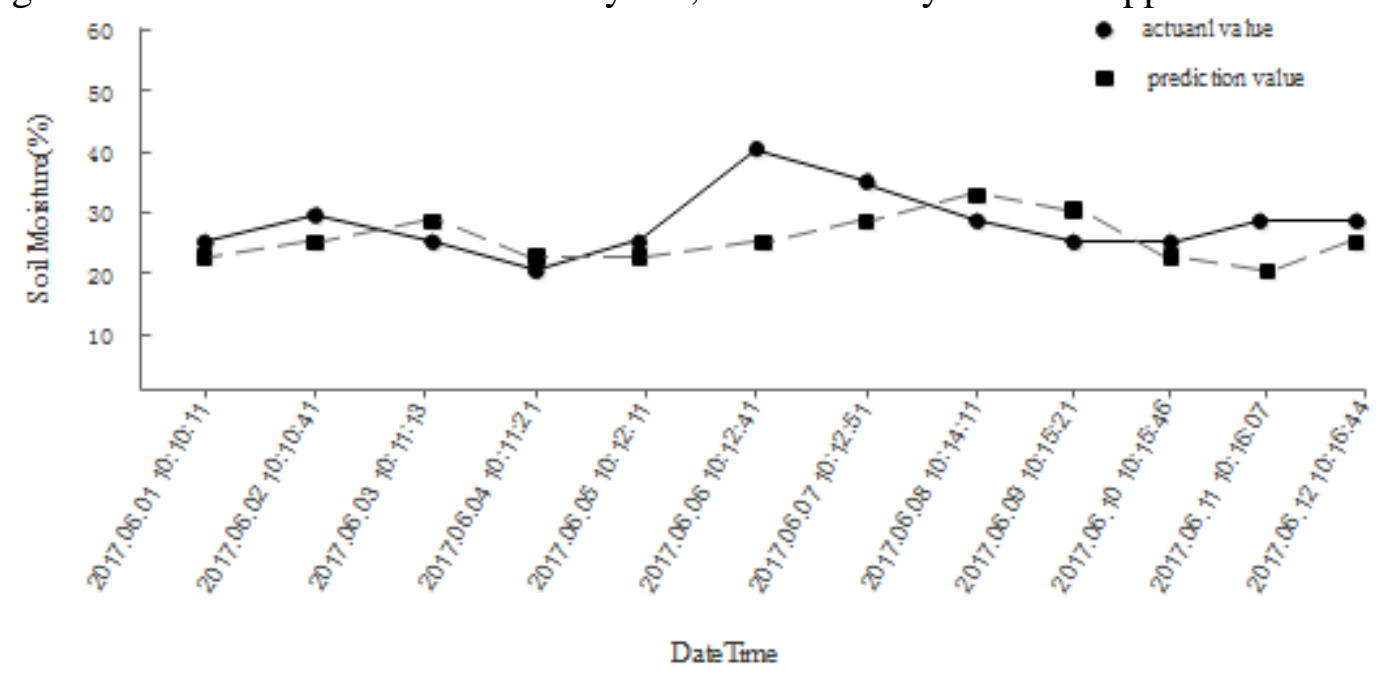

\subsection{Irrigation Policy}

Fig.6 Contrast between prediction value and actual value

Setting the threshold ${ }_{x}^{-}$of soil moisture according to water requirement of crop growth cycle. When actual value ${ }_{x}{ }^{(0)}(i)<{ }_{x}$ open the electromagnetic valve in water-saving irrigation node to irrgation. On the basis of ratio between moisture prediction value $\hat{\hat{x}}_{\hat{\hat{n}^{(0)}}}^{(i+1)}$, of next time and ${ }^{(0)}{ }_{(i)}$ to increase or decrease the amount of irrigation $\frac{-}{y} \cdot a=\frac{\hat{x}^{(0)}(i+1)}{x^{(0)}(i)}$, the amount of irrigation ${ }_{\mathrm{y}}=(1-\mathrm{a})_{\mathrm{y}}+\overline{\mathrm{y}}_{\mathrm{y}}$. The amount of irrigation $\overline{\mathrm{y}}_{\mathrm{y}}$ is calculted by agricultural experts based on the factors of soil characteristics, surface evaporation and crop water demand.

\section{Summary}

This system uses IOT technology to designed soil moisture monitoring node and water-saving node,and set up WSN in the farmland to achieved real-time value of soil moisture and realized automatic irrigation.Builded soil moisture prediction model by using grey prediction method GM(1,1).Irrigation on the result of the model prediction can satisfied crop growth demand and also saving the irrigation amount.Rainfall has great influence on soil moisture, but our model does not take the factor in consideration, so when rainfall occurred there is a big error of the model prediction.Take rainfall factor to modified u parameter of the model is our future work.

\section{References}

[1]. Yuchuan Fan, Xudong Yan, Hongfei Zhang, et al. Crops suitable evalution and water-saving irrigation mode allocation in Inner Mongolia.Journal of Agricultural Engineering.Vol. 33(2017) No. 21, p. 115-127. 
[2]. Chunsheng Zhou, Haibin Shi.Summary of reasearch on water-saving irrigation technology. Journal of Inner Mongolia University.Vol. 30(2009) No. 04,p. 314-320.

[3]. Lijun Kang, Renzhi Zhang, Lili Wu, et al. Water-saving irrigation linkage control system. Journal of Agricultural Engineering.Vol. 27(2011) No. 08,p. 232-236.

[4]. Wenfeng Li.Application of BP neural network in soil moisutre prediction model in XuChang.Chinese agricultural science bulletin.Vol. 29(2013) No. 32,p. 238-241.

[5]. Yufei Liu, Min Huang, Qibing Zhu.Design of remote monitoring system for aquaculture based on ARM_GPRS_ZIGBEE.Computer technology and development.Vol. 22(2013) No. 09, p. 181-184.

[6]. Junbing Li, Yongzhong Hu.Application design of ZigBee communication network based on CC2530.Electronic design engineering.Vol. 19(2011) No. 16,p. 108-111.

[7]. Ronghua Ji, Xin Li, Shulei Zhang, et al.Short-term prediction of soil moisture in Farmland Based on GM (1,1) model group.Journal of agricultural machinery.Vol. 10(2016) No. 47,p. 401-407.

[8]. Xiying Zhang.Crops roots and soil water usage. Meteorology Press of Beijing,1999.

[9]. Tianhua Cheng, Haitao Tang.Remote soil moisture monitoring and forecasting system based on ARM and GPRS.Journal of Agricultural Engineering.Vol. 08(2012) No. 03, p. 162-166.

[10]. Wei Yichang, Wang Zhenying, Wang Tongchao.Design of real time soil moisture monitoring and precision irrigation systems[J]. Transactions of the Chinese Society of Agricultural Engineering.2013,29(17):80-86 